Markus Illner, Erik Esche, Jens-Uwe Repke

\title{
Optimal Control of Surfactant containing Multiphase Systems - Challenges and Solution Strategies for a stable Mini-Plant Operation
}

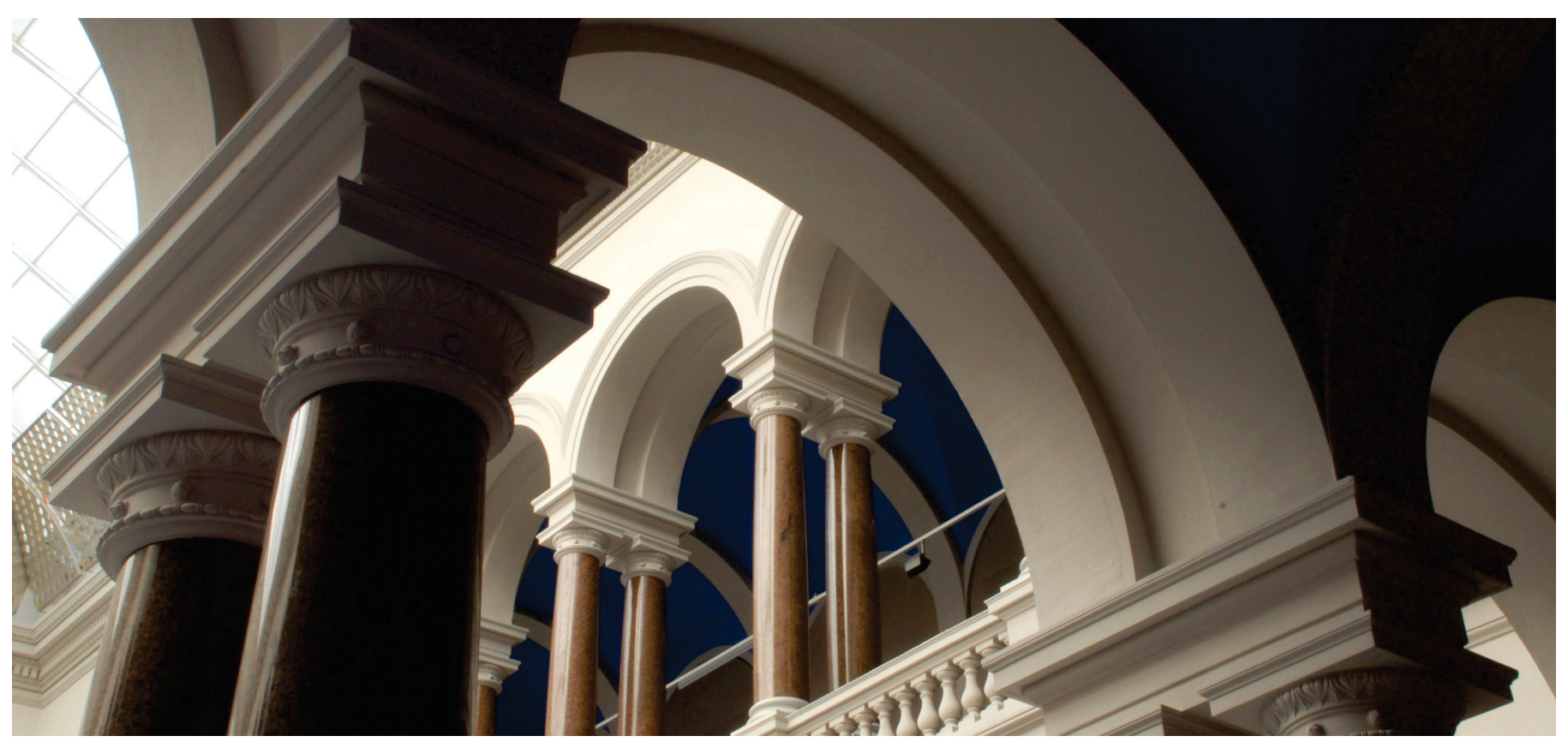

Illner, M., Esche, E., \& Repke, J.-U. (2018). Optimal Control of Surfactant containing Multiphase Systems - Challenges and Solution Strategies for a stable Mini-Plant Operation. In 13th International Symposium on Process Systems Engineering (PSE 2018). (Computer Aided Chemical Engineering ; 44) pp. 739-744. https://doi.org/10.1016/b978-0-444-64241-7.50118-x 
Mario R. Eden, Marianthi Ierapetritou and Gavin P. Towler (Editors) Proceedings of the $13^{\text {th }}$ International Symposium on Process Systems Engineering - PSE 2018

July 1-5, 2018, San Diego, California, USA (C) 2018 Elsevier B.V. All rights reserved.

\title{
Optimal Control of Surfactant containing Multiphase Systems - Challenges and Solution Strategies for a stable Mini-Plant Operation
}

\author{
Markus Illner $^{\mathrm{a}^{*}}$, Erik Esche ${ }^{\mathrm{a}}$, Jens-Uwe Repke ${ }^{\mathrm{a}}$ \\ ${ }^{a}$ Technische Universität Berlin, Process Dynamics and Operations Group, Sekr. KWT- \\ 9, Straße des 17. Juni 135, D-10623, Germany \\ markus.illner@tu-berlin.de
}

\begin{abstract}
In this contribution, a model-based approach for the control and stable operation of a mini-plant for the hydroformylation in microemulsions is presented. Within these systems, the control of the crucial reaction and phase separation steps is hindered by shifting operation windows and immeasurable concentrations. Combining the optical observation of the separation state and an underlying phase separation model, a softsensor is created to identify these relevant concentrations. Together with plant measurements, a moving horizon state estimation is used to calculate a consistent and validated state of the full plant model, which is then used in a dynamic optimization to calculate optimal trajectories for the mini-plant operation. Hereby, a stable separation is achieved for up to $200 \mathrm{~h}$ mini-plant campaigns, alongside an efficient reaction performance with up to $38 \%$ yield and an overall selectivity of $95 \%$.
\end{abstract}

Keywords: Soft-sensor, State Estimation, Microemulsion, Mini-plant.

\section{Introduction and Motivation}

In recent years, tuneable solvent systems, which especially enable new synthesis paths for the conversion of usually long-chained bio-based starting materials have emerged. Substitutionary, a surfactant containing system for the hydroformylation of longchained alkenes together with synthesis gas towards the corresponding aldehydes, using aqueous catalyst solutions is regarded within this contribution. Following Figure 1, the induced microemulsion provides a large interfacial area for increased reaction rates in a reactor. Subsequently, a gravity settler allows for an efficient separation of the products (oil phase), as well as the catalyst recycling (aqueous phase) using a gravity settler. To test this concept, a mini-plant has been built and operated (Illner et al., 2016).

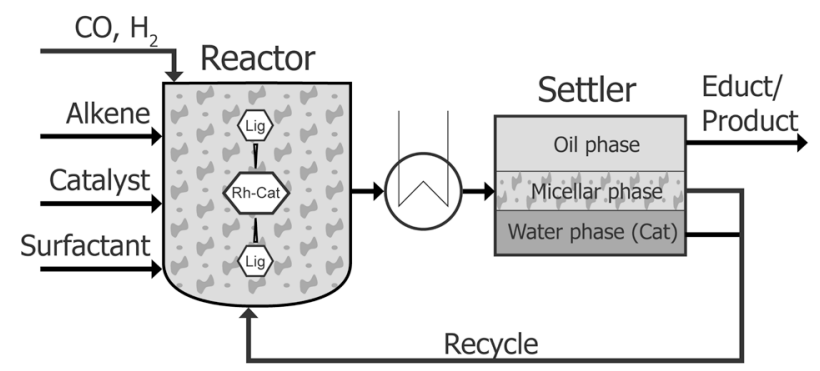

Figure 1: General process concept for the hydroformylation in microemulsions 
However, the operability of such a system remains critical due to the complexity of the component system and immeasurable states. Since standard automation approaches fail in this case, a stable operation is achieved with model-based strategies. This comprises a detailed investigation of the phase separation system, from which a soft-sensor was derived to gain relevant information on concentrations in the plant. Subsequently a state estimation can be carried out to calculate initials for the complete plant model. A dynamic real-time optimization is then used to generate optimal trajectories for the set points of the mini-plant's base automation controllers. To prove this concept, long-term min-plant campaign were carried out, aiming for a stable operation of especially the crucial phase separation and the reaction step.

\section{Background Information and Technical Application}

Investigations on the afore mentioned process concept are carried out for the exemplarily alkene 1-dodecene, the surfactant Marlipal ${ }^{\circledR} 24 / 70$, and a catalyst solution consisting of water, a rhodium precursor (CAS: 14874-82-9) and SulfoXantPhos.

\subsection{Mini-plant Set-up}

The constructed mini-plant consists of a feed section, holding feed tanks for the used components and corresponding feeds pumps, which supply a high pressure reactor, equipped with a gassing stirrer. The separation is carried out in a self-constructed settler unit augmented with gauge glasses and three drains. Thus, three developed phases can be recycled individually within the process, whereas the oil phase is also conveyed towards a product tank. The plant is fully automatized with SIEMENS PCS 7 and offline gas chromatography is used for sampling of liquids. Further information on the system, safety layers, and analytics can be found elsewhere (Illner et al., 2016).

\subsection{Separation Behaviour of Microemulsion Systems and Operation Window}

A ternary mixture of water, oil, and a surfactant, is characterized by the formation of microscopic scale structures, so called micelles. The formation of micelles, their type, and the development of excess phases is influenced by temperature and concentration (Figure 2, left). For the depiction hereof, the Kahlweit's Fish plot in Figure 2 is used.
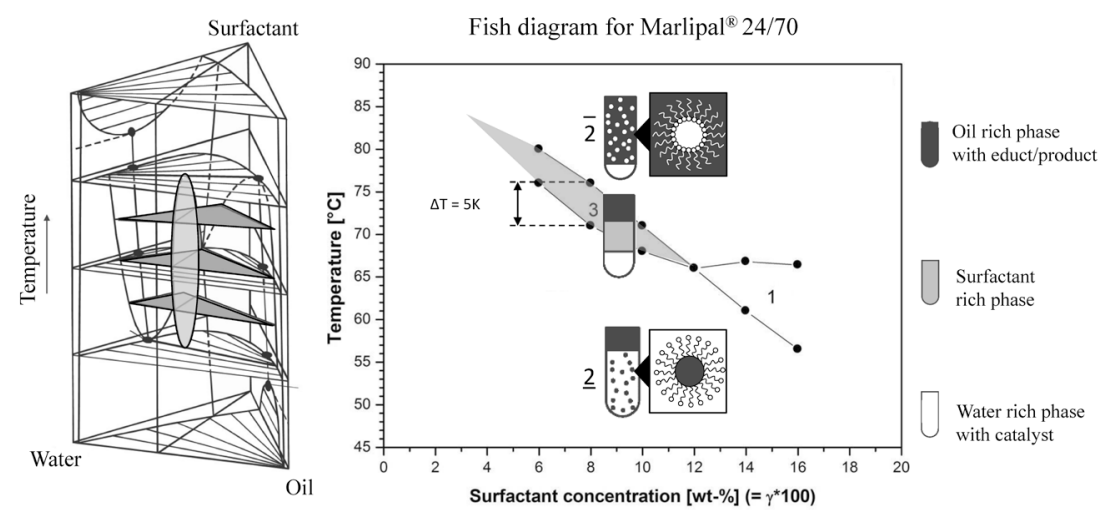

Figure 2: Left: Gibb's phase prism for oil, water, and a nonionic surfactant. Grey areas indicate three phasic miscibility gaps. Taken from (Stubenrauch et al., 2009). Right: Kahlweit's Fish plot with temperature dependent separation regimes for the regarded component system. Adapted from (Pogrzeba et al., 2017) 
Optimal Control of Surfactant containing Multiphase Systems -

Regarding the process concept, only the three-phase region (3) is feasible for the plant operation, due to the possibly high catalyst loss towards the oil phase in the $\overline{2}$-state and rather slow separation dynamics for the $\underline{2}$-state. Thus, a desired operation window for a successful phase separation is identified.

\subsection{Operation Challenges}

Controlling the phase separation state for a plant operation requires the identification of relevant influence parameters, their measurability and controllability. Table 1 hereof lists relevant process variables, directly influencing the position of the three-phase region. Besides the separation temperature, which is easily accessible and measured in sufficient accuracy, relevant influence parameters are only available with a large delay (concentration measurements) or are not accessible at all (surfactant concentration). Especially the latter hinders the controllability of the system dramatically, since also the influence on the position and expanse of the phase separation operation window is the largest (see Figure 2). Thus, frequent failures of the separation are to be expected, even with only small perturbations of a steady state operation point. Moreover, Müller (2017) and Illner (2016) reported an affection of the hydroformylation reaction through the separation state, resulting in a reduction of the selectivity towards the target product.

Table 1: Process variables influencing the phase separation step, their sensitivity on the separation operation region location, measurability, and controllability. STD: Relative standard deviation of nominal measurement value at standard operation point.

\begin{tabular}{llllll}
\hline $\begin{array}{l}\text { Process } \\
\text { variable }\end{array}$ & $\begin{array}{l}\text { Sensitivity on } \\
\text { separation }\end{array}$ & Measurability & $\begin{array}{l}\text { STD } \\
\text { in } \%\end{array}$ & $\begin{array}{l}\text { Sampling } \\
\text { frequency }\end{array}$ & Controllability \\
\hline Temperature & high & $\begin{array}{l}\text { Temperature } \\
\text { sensors }\end{array}$ & 0.003 & $1 \mathrm{~s}$ & Heater \\
$\begin{array}{l}\text { Oil to water } \\
\text { ratio }\end{array}$ & moderate & $\begin{array}{l}\text { Offline gas } \\
\text { chromatography }\end{array}$ & 0.7 & $1 \mathrm{~h}$ & Feed pump \\
$\begin{array}{l}\text { Product } \\
\text { concentration }\end{array}$ & moderate & $\begin{array}{l}\text { Offline gas } \\
\text { chromatography }\end{array}$ & 0.7 & $1 \mathrm{~h}$ & $\begin{array}{l}\text { Feed pump, } \\
\text { residence time }\end{array}$ \\
$\begin{array}{l}\text { Surfactant } \\
\text { concentration }\end{array}$ & very high & Not available & - & - & - \\
\hline
\end{tabular}

\section{Model Development and Control Strategy}

An approach to enable the controllability and stable operation of the hydroformylation process in microemulsions is to develop a model-based description of the phase separation behaviour and derive a soft-sensor for relevant concentrations herefrom. To additionally account for the highly dynamic and non-steady state process behaviour, the development of operation trajectories from a dynamic optimization is employed.

\subsection{Phase Separation Model and Surfactant Soft-Sensor}

Since no generic thermodynamically driven description of microemulsion systems is available, an empiric model is created based on experimental data. The main idea is to exploit the separation characteristics for the desired three-phase region (3). For constant temperature $\mathrm{T}$ and pressure $\mathrm{p}$, the mixture of oil, water, and surfactant, separates into three phases with certain compound concentrations $c_{i}^{\text {Phase }}$. This corresponds to the corners of the triangle miscibility gap in the ternary diagram (see dark grey area in the 
Gibb's phase prims in Figure 2). If the mixture concentration is varied, the same individual phases with an equal composition will occur, but the volume fractions will be shifted. Thus, a correlation between the compound concentrations in the initial mixture $c_{i}^{\text {Mixture }}$, the volume fractions $\varphi^{\text {Phase }}$ and compound concentrations $c_{i}^{\text {Phase }}$ is derived:

$c_{i}^{\text {Mixture }}(T, p)=f\left(c_{i}^{\text {Phase }}(T, p), \varphi^{\text {Phase }}(T, p)\right)$

Given experimental results on $\varphi^{\text {Phase }}$ from a full factorial design of experiments over the relevant influence factors according to Table 1, Equation 1 is transferred into a polynomial model with $\varphi^{\text {Phase }}, \mathrm{T}$, and $\mathrm{p}$ as inputs and fixed parameters $c_{i}^{\text {Phase }}$ and $P_{n}$. Additionally, the component concentrations $c_{i}^{\text {Phase }}$ are gathered using offline gas chromatography measurements as well as determination of density and the critical micelle formation concentration, which represents the surfactant concentration in the oily and aqueous excess phase.

Subsequently, the surfactant concentration in the settler becomes accessible with a softsensor. Combining the optical observation of the separation performance through gauge glasses via a webcam with an automated image processing results in frequent information on developed phase heights in the settler. Together with the geometric information of this unit, $\varphi^{\text {Phase }}$ is calculated and used together with the settler temperature measurement to identify the settler mixture concentrations $c_{i}^{\text {Mixture }}$

\subsection{Calculation of optimal trajectories}

With this approach, the first-time online identification of the relevant surfactant concentration for the microemulsion system at hand is possible and enables further control strategies in regard to dynamic optimization. Therefor, a discretized dynamic mini-plant model described by Müller et al. (2017) is used. As a first step, measurement data from the mini-plant, offline gas chromatography sampling and the surfactant soft sensor is collected and processed in a data conciliation and moving horizon state estimation framework. Extending the work of Hoffman et al. (2016) for dealing with gross errors, the handling of different sampling rates (see Table 1) is incorporated here. This is done by first solving the optimization problem for a reduced plant model containing only component mass balances to estimate stream and level information. Subsequently, when additional slow concentration measurements are available, to full problem is solved and updated. Gaining validated initials of the full mini-plant model, a dynamic optimization is then carried out. The objective is to maximize the reaction product content in the product stream within the next $4 \mathrm{~h}$. Constraints apply on the reactor and settler surfactant concentration and oil to water ratio in order to maintain an efficient reaction and a stable separation. This results in optimal trajectories for the controller set points of feed streams, recycle streams, and settler temperature.

\section{Application on Mini-plant Operation}

A mini-plant operation is conducted for a total of $180 \mathrm{~h}$, applying several operation modes. The startup is realized by gravimetrically feeding the required substances and firstly initializing a full recycle operation. Afterwards the reaction is started with the introduction of synthesis gas. The optimizer is started in parallel and calculated trajectories are applied manually in the process control system. 
Optimal Control of Surfactant containing Multiphase Systems -

\subsection{Separation performance}

The overall separation performance is evaluated by the amount of oily substances (reaction educt and products) in the settler oil phase, as well as the catalyst leaching. Here, an excellent separation is achieved with a mean oil phase purity of $>99.5 \%$ and a leaching of the rhodium catalyst below $0.1 \mathrm{ppm}$. Additionally, the effect of the applied optimization and soft-sensor approach is depicted in Figure 3. For a reference time frame of $2 \mathrm{~h}$, the separation performance is observed via a gauge glass. The corresponding evaluation of the soft-sensor implies a reduction of the surfactant concentration, which consequently established with a reduction of the middle emulsion phase fraction. As a control action, the optimizer calculation the addition of surfactant to retain the desired operation point.

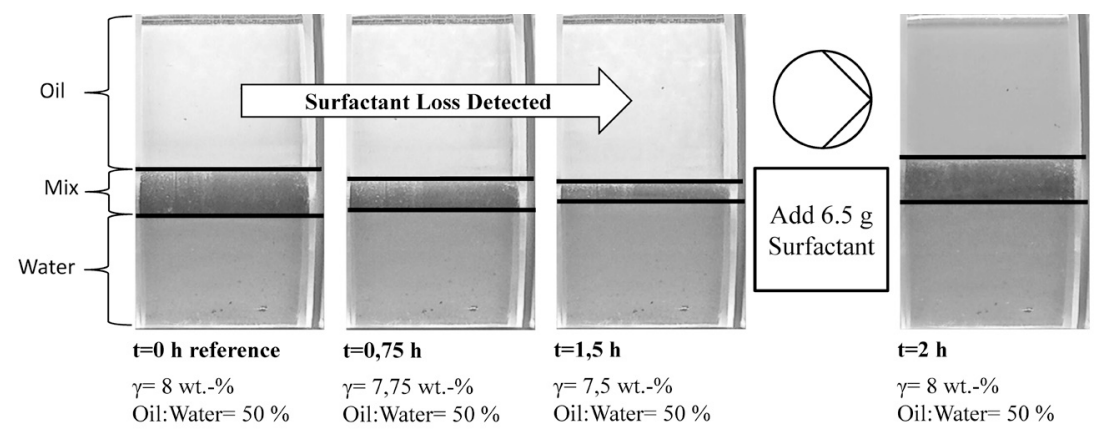

Figure 3: Gauge glass images of the settler over an operation horizon of $2 \mathrm{~h}$ and results of the soft-sensor calculations. The right picture shows the separation state after a performed control action derived from the dynamic optimization.

\subsection{Reaction}

Figure 4 shows the reaction performance for the applied reaction residence time of $7 \mathrm{~h}$, whereas a steady state conversion of the applied alkene 1-dodecene of $38 \%$ is achieved. Moreover, a chemoselectivity towards the main product tridecanal of $95 \%$. This meets results from reference lab scale investigations and shows a successful transfer of the process concept in to the mini-plant.

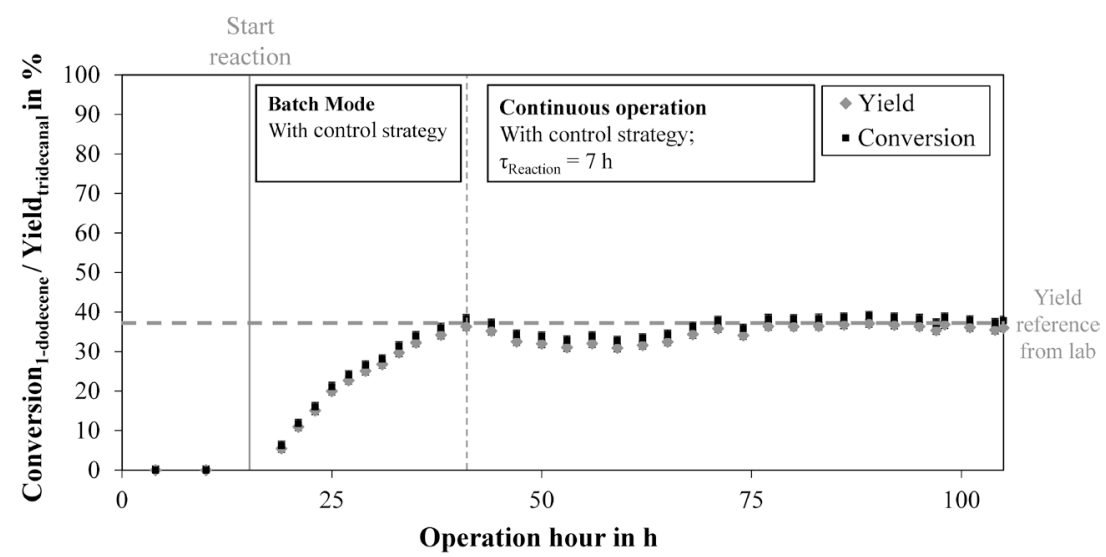

Figure 4: Mini-plant operation results for the conversion of 1-dodecene and the yield of the main product tridecanal for different operation modes. 


\section{Conclusions and Outlook}

Within this contribution, a model-based approach has been presented to cope with the highly dynamic and non-steady state process behaviour of a hydroformylation process in microemulsions. Herein, an empiric model for the separation state and a soft-sensor for the identification of immeasurable surfactant concentrations are used to calculate valid initials for a full mini-plant model. Using calculated trajectories from a dynamic optimization, controller set-point of the process control system a stable mini-plant operation with successful reaction and separation could be achieved.

Future works will focus on the online application of the represented framework, using OPC UA to directly interface the process control system. Additionally, the state estimator is to be extended towards a simultaneous handling of multi sampling rates. Finally, further mini-plant operations will focus on testing the robustness of the used optimization framework regarding multiple or large perturbations.

\section{Acknowledgements}

This work is part of the Collaborative Research Centre 'Integrated Chemical Processes in Liquid Multiphase Systems' (subproject B4/C4) coordinated by the Technical University of Berlin. Financial support from the German Research Foundation (Deutsche Forschungsgemeinschaft, DFG) is gratefully acknowledged (TRR 63). Furthermore, the authors gratefully acknowledge the support of Umicore N.V. for sponsoring the rhodium catalyst precursor 'Acetylacetonatodicarbonylrhodium(I)' (CAS: 14874-82-9), Sasol Ltd. for the surfactant used in the described experiments, the support of SIEMENS AG for sponsoring the entire process control system, SIMATIC PCS7 for the automation of the mini-plant, and Rhodius $\mathrm{GmbH}$ for sponsoring the knitted fabrics.

\section{References}

C. Hoffmann, M. Illner, D. Müller, E. Esche, L. T. Biegler, G. Wozny, 2016, Moving-horizon State Estimation with Gross Error Detection for a Hydroformylation Miniplant, Computer Aided Chemical Engineering, 38, 1485-1490.

M. Illner, D. Müller, E. Esche, T. Pogrzeba, M. Schmidt, R. Schomäcker, G. Wozny, J.-U. Repke, 2016, Hydroformylation in microemulsions: proof of concept in a mini-plant, Industrial \& Engineering Chemistry Research, 55, (31), 8616 - 8626.

M. Müller, Y. Kasaka, D. Müller, R. Schomäcker, G.Wozny, 2013, Process Design for the Separation of Three Liquid Phases for a Continuous Hydroformylation Process in a Miniplant Scale, Industrial \& Engineering Chemistry Research, 53, 7259 - 7264.

D. Müller, M. Illner, E. Esche, T. Pogrzeba, M. Schmidt, R. Schomäcker, L. Biegler, G. Wozny, J.-U. Repke, 2017, Dynamic real-time optimization under uncertainty of a hydroformylation mini-plant, Computers and Chemical Engineering, (2017), 108, 836-848.

T. Pogrzeba, M. Schmidt, N. Milojevic, C. Urban, M. Illner, J.-U. Repke, R. Schomäcker, 2017, Understanding the Role of Nonionic Surfactants during Catalysis in Microemulsion Systems on the Example of Rhodium-Catalyzed Hydroformylation, Industrial \& Engineering Chemistry Research, 56 (36), $9934-9941$.

C. Stubenrauch, 2009, Microemulsions: Background, New Concepts, Applications, Perspectives, John Wiley \& Sons, Ltd: Chichester, UK 